\title{
Sema4C modulates the migration of primary tumor-associated lymphatic endothelial cells via an ERK-mediated pathway
}

\author{
JIN PENG ${ }^{1 *}$, XIJIANG LIU ${ }^{2 *}$, CHENGCHENG LI ${ }^{1}$, \\ $\mathrm{MIN} \mathrm{GAO}^{1}$ and HONGYAN WANG ${ }^{1}$ \\ ${ }^{1}$ Department of Obstetrics and Gynecology, Qilu Hospital, Cheeloo College of Medicine, Shandong University, \\ Jinan, Shandong 250001; ${ }^{2}$ Department of Anesthesiology, Shandong Provincial Hospital Affiliated \\ to Shandong First Medical University, Jinan, Shandong 250021, P.R. China
}

Received November 17, 2020; Accepted June 18, 2021

DOI: $10.3892 / \mathrm{etm} .2021 .10535$

\begin{abstract}
Although lymphatic endothelial cells (LECs) serve a positive role in tumor lymphatic metastasis, the regulation of LECs undergoing migration similar to that of tumor cells remains poorly understood. A previous study revealed that semaphorin 4C (Sema4C) could be a marker of LECs in cervical cancer. Thus, the present study aimed to understand the mechanism via which Sema4C could promote the development of tumor-associated characteristics in LECs in cervical cancer. Primary tumor-associated LECs (TLECs) were distinguished from cervical cancer by flow cytometry. The promigratory ability was assessed using the Transwell assay. Lentivirus infection was used to alter the expression of Sema4C in TLECs. Confocal laser scanning was used to determine the infection efficiency of lentivirus infection. Sema4C/ERK/E-cadherin pathway was measured by reverse transcription-quantitative PCR and western blotting. The co-localization of Sema4C and the lymphatic marker lymphatic vessel endothelial hyaluronan receptor 1 was verified. Primary tumor-associated LECs (TLECs) were isolated from a mouse xenograft cervical tumor model. It was revealed that overexpressing Sema4C stimulated the migratory ability of TLECs, downregulated E-cadherin expression and stimulated ERK phosphorylation, whereas knocking down Sema4C had the opposite effects. The treatment of PD98059 (ERK inhibitor) blocked the pro-migratory ability of TLECs, which indicated a dependence on the ERK signaling pathway. It was identified that the Sema4C/ERK/E-cadherin pathway may be critical
\end{abstract}

Correspondence to: Professor Hongyan Wang, Department of Obstetrics and Gynecology, Qilu Hospital, Cheeloo College of Medicine, Shandong University, 107 West Wenhua Road, Jinan, Shandong 250001, P.R. China

E-mail: hongyanwang@sdu.edu.cn

*Contributed equally

Key words: semaphorin 4C, tumor-associated lymphatic endothelial cells, E-cadherin, extracellular signal-regulated kinase for the migration of TLECs, which may promote lymph node metastasis. Therefore, Sema4C could be a promising target for the treatment of cervical cancer with lymphatic metastasis.

\section{Introduction}

Lymphatic metastasis is one of the crucial routes of metastasis in cervical cancer (1) and treatment failure in cervical cancer is often associated with lymph node metastasis (2). Lymphatic vessels are usually considered to serve a passive role in metastasis, acting merely as a channel for tissue-invading tumor cells (3). Previous studies have focused on the immunoregulatory function of lymphatic endothelial cells (LECs), which are active players in lymphatic metastasis $(3,4)$. However, it remains unknown whether and how LECs actively regulate lymphatic metastasis in cancer.

Endothelial-mesenchymal transition (EndMT) is a process via which endothelial cells (ECs) display considerable plasticity in the transition to mesenchymal cells, which is crucial for ECs to obtain migratory abilities similar to those of tumor cells $(5,6)$. During this process, ECs lose their endothelial markers, such as E-cadherin and CDs, and acquire a migratory phenotype and mesenchymal markers, such as vimentin and fibroblast-specific protein $1(5,6)$. LECs also express E-cadherin and can be induced to enter endothelial-mesenchymal transition (EndMT) via WNT5B and Kaposi's sarcoma-associated herpesvirus $(7,8)$. E-cadherin is a major component of epithelial cell junctions, as well as a hallmark of EndMT $(6,9)$. However, the current understanding of the regulatory mechanism underlying E-cadherin expression in EndMT remains limited. In EMT, which is analogous to EndMT, epidermal growth factor is suggested to induce the downregulation of E-cadherin via the ERK pathway (10), which may activate a similar signaling pathway in EndMT.

Semaphorins (Semas) are a large family of extracellular signaling proteins that regulate the motility of cells during the development of nevi (Sema3A, 3F, 6C and 6D), the neuroendocrine system (Sema7A and 4D), the immune system (Sema4D), the reproductive system (Sema3) and cancer progression (Sema4D, 3A and 6D) (11-13). Interestingly, Semas antagonize the effect of VEGF (13). In addition, several studies have reported that Sema3F may affect lymphangiogenesis $(14,15)$. 
In a previous study, tumor-associated LECs (TLECs) were isolated and normal LECs were obtained from tissues using in situ laser capture microdissection, and it was determined that synthetic membrane-bound Sema4C functioned as an autocrine factor that promoted lymphangiogenesis (16). However, the underlying mechanism of Sema4C in regulating TLEC biological characteristics is largely unknown.

It has been revealed that tumors or metastasis may rely, at least in part, on the gene regulatory events in cells that constitute the primary tumor, as well as the tumor microenvironment (TME) (17). LECs, which are one of the most important components in the TME, also exhibit a unique gene expression pattern compared with non-tumor LECs, such as the expression of VEGFR3, which is activated under pathological conditions, including cancer and inflammation (18). LECs bought from ScienCell ${ }^{\mathrm{TM}}$ (cat. no. 2500) are separated from human lymph nodes, according to the instructions of the cell line. The differential expression level of genes between normal LECs and TLECs prompted the present study to assess TLECs from tumors. Flow cytometry has been indicated to be the most effective method for isolating cells, as well as analyzing the TME $(19,20)$. To the best of our knowledge, the present study for the first time used primary TLECs separated from mouse cervical tumors to study LEC biological characterization.

The present study evaluated the role of Sema4C in the transdifferentiation of primary TLECs to determine the tumor cell-like invasive characteristics. The aim of the present study was to investigate the effect of Sema4C on the migration of primary TLEC isolated from tumor tissues by flow cytometry and its mechanism using lentivirus infection to modulate the expression of Sema4C. These findings indicated that regulation of the Sema4C/ERK/E-cadherin pathway may be a novel target for cancer therapy, which may potentially inhibit endothelial transdifferentiation into tumor-like cells, thereby preventing the lymphatic metastasis of cancer. The current preclinical study provided novel insights into the role of Sema4C in TLECs and demonstrated the active participation of TLECs in lymphatic metastasis.

\section{Materials and methods}

Antibodies and cell line. All reagents used in the present study were of analytical grade and are commercially available. The primary antibodies used were as follows: Sema4C (cat. no. sc-136445; Santa Cruz Biotechnology, Inc.); lymphatic vessel endothelial hyaluronan receptor 1 (LYVE1) antibody-Alexa Fluor ${ }^{\circledR} 488$ for flow cytometry (cat. no. 53-0433-82; eBioscience; Thermo Fisher Scientific, Inc.); LYVE1 antibody for immunohistochemistry (cat. no. ab14917; Abcam); VEGFR3 (cat. no. ab51874; Abcam); E-cadherin (cat. no. ab181296; Abcam); ERK1/2 (cat. no. ab17942; Abcam), phosphorylated (p)-ERK1/2 (cat. no. ab214362; Abcam) and $\beta$-actin (cat. no. ab8227; Abcam). Biotinylated anti-rabbit immunoglobulin for DAB staining (cat. no. K406511-2) was purchased from Agilent Technologies, Inc. The ERK inhibitor PD98059 (cat. no. HY-12028) was purchased from MedChemExpress and cells were treated with $30 \mathrm{mM}$ for $24 \mathrm{~h}$ at room temperature.
The mouse cervical cancer cell line U14 (cat. no. YB-M060) was purchased from Shanghai Yubo Biotechnology Co., Ltd. The cells were cultured in RPMI-1640 medium (cat. no. 21870076; Gibco; Thermo Fisher Scientific, Inc.) $37^{\circ} \mathrm{C}$ with $5 \% \mathrm{CO}_{2}$.with $10 \%$ (v/v) FBS (cat. no. 16140071; Gibco; Thermo Fisher Scientific, Inc.) NIH3T3 cells (mouse embryonic fibroblast cell line) was purchased from ATCC (cat. no. CRL-1658), cultured in Dulbecco's Modified Eagle's Medium (cat. no. 11885084; Gibco; Thermo Fisher Scientific, Inc.) with $10 \%$ (v/v) FBS (cat. no. 16140071; Gibco; Thermo Fisher Scientific, Inc.) at $37^{\circ} \mathrm{C}$ with $5 \% \mathrm{CO}_{2}$. No antibiotics were used in the culture medium. The use of primary LECs was approved by the Scientific Research Ethics Committee of Shandong University.

Lentivirus for delivery of full-length Sema4C and small interfering (si)RNA against Sema4C. All the lentiviruses used in the present study were purchased from Wester Biological Technology Co.,Ltd.(serial no.WST201710015).Each lentiviral packaging system included a four-plasmid system (Fig. S1). A full-length mouse Sema4C open reading frame was obtained via PCR (PrimeSTAR ${ }^{\circledR}$ HS DNA Polymerase; cat. no. R010A; Takara BioInc.) (thermocycling conditions were as follows: $95^{\circ} \mathrm{C}$ for $3 \mathrm{~min} 1$ cycle, followed by 30 cycles of $94^{\circ} \mathrm{C}$ for $30 \mathrm{sec}$, $-55^{\circ} \mathrm{C}$ for $40 \mathrm{sec}$ and $-72^{\circ} \mathrm{C}$ for $30 \mathrm{sec}$ and $72^{\circ} \mathrm{C}$ for $5 \mathrm{~min}$ for 1 cycle), using cDNA (from TLECs) by agarose electrophoresis and gel-cutting recovery as template (cat. no. DP219-03; Tiangen Biotech Co. Ltd.) and the primer sequences were as follows: Forward, 5'-AGGGTTCCAAGCTTAAGCGGCCGC GCCACCATGGCCCCACACTGGGCTGTCTGG-3' and reverse, 5'-GATCCATCCCTAGGTAGATGCATTCATACT GAAGACTCCTCTGGGTTG-3'. The lentivirus used for delivery of full-length Sema4C was LV5. The final construction was termed LV5-Sema4C. The control construction was LV5 negative control (empty vector, LV5NC).

The lentivirus used for delivery of siRNA against Sema4C was LV3. The Sema4C siRNA sense sequence was 5'-CCU AUGCCUUCCAGCCCAADTDT-3' and antisense sequence was 5'-UUGGGCUGGAAGGCAUAGGDTDT-3', and the final construction was termed LV3-siRNA. The sequence for the control siRNA was a non-targeting sequence, sense, 5'-UUCUCCGAACGUGUCACGU-3' and antisense, 5'-ACG UGACACGUUCGGAGAA-3', and the lentiviral construct was termed LV3NC. The Sema4C siRNA has been verified in a previous study (10).

Animals. C57BL/6 female mice (age, 4-6 weeks; weight, 18-22 g) were purchased from Tengxin Biomedical Technology. The mice were maintained in the accredited animal facility of Shandong University. The animal facility had a controlled temperature $\left(23-25^{\circ} \mathrm{C}\right)$, a $12 / 12 \mathrm{~h}$ light/dark cycle with lights on from $8: 00$ to $20: 00$, and a relative humidity (50-60\%). The mice had free access to standard food and water. Animals were cared for by qualified personnel every day, including weekends and holidays, to monitor their health and behavior. The hygienic status of the mice was specific pathogen-free, according to the Association for Assessment and Accreditation of Laboratory Animal Care International (Laboratory Animal Center of Tsinghua University) recommendations. Each group contained 3 mice, and there were three time points (days 5, 7 
and 14 after the injection of U14 cells) and 9 mice in total No mouse died until the end of the experiments. All the experiments were carried out in accordance with the National Institute of Health Guide of the Care and Use of Laboratory Animals. All the experimental protocols were approved by the Animal Care and Use Committee of Shandong University for animal ethics [approval no. KYLL-2015(KS)-079].

Mouse xenograft tumor model. The mice were anesthetized using isoflurane $/ \mathrm{O}_{2}$ flow (4\% induction and $2 \%$ maintenance dose) in an induction chamber. Forceps were used to touch the leg of the mouse, which was used to confirm that the mouse was fully unconscious. The mice received $100 \mu 1\left(\sim 1 \times 10^{7}\right)$ U14 cells, resuspended in RPMI-1640 medium, subcutaneously into the left shoulder. A total of 2 weeks after the injection, the animals was euthanized and the tumor volume was calculated according to the following formula: Length $x$ width $2 / 2$ (21). The cage was placed in a chamber with $\mathrm{CO}_{2}$, and $\mathrm{CO}_{2}$ was turned on for $\geq 20 \mathrm{~min}$ at $2 \mathrm{l} / \mathrm{min}$. The percentage of the chamber volume/min displaced by the flow rate of $\mathrm{CO}_{2}$ was $20 \%$ (April 2019). $\mathrm{CO}_{2}$ was maintained for $\geq 1 \mathrm{~min}$ after the mice stopped breathing. The sacrificed mice were placed on a polystyrene board, and the tumor was removed and measured.

Separation of TLECs via flow cytometry. Tumor tissue cells were dissociated using 1\% collagenase (cat. no. C2139; Sigma-Aldrich; Merck KGaA) for $30 \mathrm{~min}$ at $37^{\circ} \mathrm{C}$ and $0.25 \%$ trypsin/EDTA solution, (cat. no. 25200056; Gibco; Thermo Fisher Scientific, Inc.) for 5-8 $\mathrm{min}$ at room temperature. Cell suspensions were filtered through cell sieves (200- $\mu \mathrm{m}$ mesh). After incubation for $48 \mathrm{~h}$ at $37^{\circ} \mathrm{C}$ with $5 \% \mathrm{CO}_{2}$, the suspended cells were removed and incubated with a monoclonal antibody against mouse LYVE1-Alexa Fluor 488 or IgG1 kappa Isotype Control-Alexa Fluor 488 (1:25; cat. no. 53-4714-80; EBioscience; Thermo Fisher Scientific, Inc.) at room temperature for $30 \mathrm{~min}$, after adjusting the cell density to $5-8 \times 10^{6} / \mathrm{ml}$. The samples were analyzed on a FACSCalibur apparatus (BD Biosciences) and cells that expressed LYVE1 were isolated. LECs were cultured in EBM-2 medium (Lonza Group Ltd.) with 2\% (v/v) FBS (Gibco; Thermo Fisher Scientific, Inc.) at $37^{\circ} \mathrm{C}$ with $5 \% \mathrm{CO}_{2}$.

Immunohistochemistry for the quantification of lymphatic microvessel density (LMVD). Immunohistochemical analysis of LYVE1 was performed using the avidin-biotin-peroxidase complex method. Fresh tissue was fixed by $4 \%$ paraformaldehyde for $24 \mathrm{~h}$ and embedded using paraffin at room temperature. Dewaxed and rehydrated 5- $\mu \mathrm{m}$ mouse tumor tissue sections were incubated with $3 \% \mathrm{H}_{2} \mathrm{O}_{2}$ for $10 \mathrm{~min}$ at room temperature and then rinsed with PBS for 3 times. Sections were blocked with 5\% BSA (w/v) in PBS (cat. no. A1993; MilliporeSigma) for $20 \mathrm{~min}$ at room temperature and with a rabbit polyclonal anti-mouse LYVE1 antibody (1:100 dilution) or with PBS as the negative control overnight at $4^{\circ} \mathrm{C}$. The detection system was Dako LSAB KIT (cat. no. K406511-2; Agilent Technologies, Inc). Sections were then washed with PBS and biotinylated anti-rabbit immunoglobulin (1:200) was added to the sections for $30 \mathrm{~min}$ at room temperature. Peroxidase-conjugated avidin (1:400) was then applied after the sections were washed with PBS. The peroxidase activity was detected by exposing the sections to a solution of $0.05 \% 3,3^{\prime}$-diaminobenzidine and $0.01 \% \mathrm{H}_{2} \mathrm{O}_{2}$ in Tris- $\mathrm{HCl}$ buffer (3,3'-diaminobenzidine solution) for $10 \mathrm{~min}$ at room temperature. The sections were counterstained with hematoxylin for $30 \mathrm{sec}$ at room temperature. The stained sections were then analyzed using standard light microscopy (Eclipse 200; Nikon Corporation). Under a low magnification, the most vascularized intratumoral areas were selected (hot spots). The number of immunostained lymphatic vessels observed in three hot spot areas at x400 magnification was counted. LMVD was expressed as the mean value (total number of vessels in three hot spot microscopic fields/3).

Confocal microscopy imaging. Frozen tissue sections $(5-\mu \mathrm{m}$, solidified in liquid nitrogen) were blocked with 5\% BSA (w/v) in PBS for $20 \mathrm{~min}$ at room temperature. The LYVE1 (1:50) or Sema4C (1:20) primary antibodies were applied to the slides at $4^{\circ} \mathrm{C}$ overnight. After incubation with the primary antibodies, the slides were washed three times with cold PBS and incubated with FITC-conjugated goat anti-mouse (cat. no. A5608; Beyotime Institute of Biotechnology) or Cy3-conjugated goat anti-rabbit IgG (cat, no. A0516; Beyotime Institute of Biotechnology) at a 1:50 dilution in PBS for $30 \mathrm{~min}$ at room temperature. The nuclei were stained for $5 \mathrm{~min}$ at room temperature with DAPI. The slides were rinsed with PBS and observed using a confocal microscope and 3 fields for analyzed per sample (Olympus Corporation) (magnification, x600).

Immunohistochemistry for identification of TLECs. Immunohistochemical identification analysis of TLECs was performed using the avidin-biotin-peroxidase complex method. TLECs were incubated on coverslips $\left(2 \times 10^{4} / \mathrm{ml}\right)$ in a 12 -well plate and were fixed with $4 \%$ paraformaldehyde for $20 \mathrm{~min}$ at room temperature. The cells were blocked with $5 \%$ BSA (cat. no. A1993; MilliporeSigma w/v) in PBS for $60 \mathrm{~min}$ at room temperature, and then incubated overnight at $4^{\circ} \mathrm{C}$ with monoclonal anti-mouse VEGFR3 antibody (1:30) and then washed with PBS. Goat-anti-rat HRP-immunoglobulin (cat. no. ab97057; Abcam) was then added to the sections for $30 \mathrm{~min}$ at $37^{\circ} \mathrm{C}$. After the sections were washed with PBS, peroxidase-conjugated avidin (Dako; Agilent Technologies, Inc.) was applied. The peroxidase activity was detected by exposing the sections to a 3,3'-diaminobenzidine solution for $10 \mathrm{~min}$ at room temperature. The sections were counterstained with hematoxylin at $5 \mathrm{~min}$ at room temperature and analyzed using an inverted light microscope (Olympus Corporation) (magnification, x100).

Cell transduction. The primary TLECs were cultured in 24-well tissue culture plates or flasks at $37^{\circ} \mathrm{C}$ with $5 \% \mathrm{CO}_{2}$ in a humidified incubator (Heraeus Group). For the lentiviral infection, the cells $\left(10^{5} / \mathrm{ml} /\right.$ well; $\left.200 \mu \mathrm{l}\right)$ were incubated with lentivirus $(\mathrm{MOI}=40)$ when the cells were $70 \%$ confluent, according to the preliminary experiment. The cells were infected for $72 \mathrm{~h}$ for the subsequent experiments. The package system was a four-plasmid system, including LV5-GFP/ LV3-GFP, PG-p1-VSVG, PG-P2-REV and PG-P3-RR, purchased from Wester Biological Technology Co., Ltd. The results of transduction were detected using a confocal microscope (Olympus Corporation; magnification, x100). 
Reverse transcription-quantitative $(R T-q) P C R$. For RT-qPCR, total RNA was isolated from transduced TLECs using TRIzol ${ }^{\circledR}$ reagent (Invitrogen; Thermo Fisher Scientific, Inc.). First-strand cDNA synthesis was performed via RT using a Transcriptor First Strand cDNA synthesis kit (Amersham; Cytiva) according to the manufacturer's instructions. qPCR was performed on an ABI PRISM 7700 Sequence Detection system (Applied Biosystems; Thermo Fisher Scientific, Inc.) using a QuantiTect SYBR Green kit (Qiagen, Inc.). The primers were designed using Primer Express 3.0 software (Applied Biosystems; Thermo Fisher Scientific Inc.), as identified using Basic Local Alignment Search Tool (http://www.ncbi.nlm.nih. gov/BLAST), and were purchased from Invitrogen (Thermo Fisher Scientific, Inc.). Each sample was run in triplicate. The conditions for the qPCR reaction were as follows: One cycle at $50^{\circ} \mathrm{C}$ for $15 \mathrm{~min}$; one cycle at $94^{\circ} \mathrm{C}$ for $4 \mathrm{~min}$; and 35 cycles at $94^{\circ} \mathrm{C}$ for $20 \mathrm{sec}, 60^{\circ} \mathrm{C}$ for $30 \mathrm{sec}$ and $70^{\circ} \mathrm{C}$ for $35 \mathrm{sec}$. At the end of the PCR reaction, the samples were subjected to a melting curve analysis to confirm the specificity of the amplification. The Sema4C primer sequences were: Forward, 5'-CCTCCC ATCTGTATGTCTGCG-3' and reverse, 5'-GCTGGGTCA TATGGGCATTTAC-3'. E-cadherin, forward 5'-TCCTTG GGTGGTCTTGAT-3' and reverse 5'-CATATCGCTGTTCTT CGTT-3'; ERK 1/2 forward, 5'-TCACACAGGGTTCTTGAC AG-3' and reverse 5'-GGAGATCCAAGAATACCCA-3'. For the internal standard, the following $\beta$-actin primer sequences were used: Forward, 5'-GAGACCTTCAACACCCCAGC-3' and reverse, 5'-ATGTCACGCACGATTTCCC-3'. The method of quantification used was $2-^{\Delta \Delta C q}(22)$.

Immunoblotting. For western blot analyses, the TLEC cells treated with lentivirus or/and PD98059 were lysed in RIPA buffer (cat. no. P0013C; Beyotime Institute of Biotechnology) [50 mM Tris/HCl, pH 7.2, 150 mM NaCl, 1\% NP-40, 0.1\% SDS and $0.5 \%(\mathrm{w} / \mathrm{v})$ sodium deoxycholate]. Equivalent amounts of the cell extracts (BCA method, $20 \mu \mathrm{g}$ ) were separated via $10 \%$ SDS-PAGE and transferred onto a PVDF membrane. The membranes were blocked in $25 \mathrm{mM}$ Tris ( $\mathrm{pH}$ 8.0) containing $125 \mathrm{mM} \mathrm{NaCl}, 0.1 \%$ Tween-20 and 5\% skimmed milk for $1 \mathrm{~h}$ at room temperature, and then incubated with the diluted primary antibodies (Sema4C, 1:500; E-cadherin, ERK1/2 and p-ERK1/2, all 1:2,000; $\beta$-actin, $1: 1,000)$ at $4{ }^{\circ} \mathrm{C}$ overnight. After incubation with the primary antibodies, the alkaline phosphatase-conjugated anti-rabbit (cat. no. A9919; Sigma-Aldrich; Merck KGaA) and anti-mouse (cat. no. A4312; Sigma-Aldrich; Merck KGaA) were added at a 1:1,000 dilution. The immunoreactive bands were visualized using the ECL western blotting technique. The software used for densitometry was Image J v.1.8.0 (National Institutes of Health).

Migration assay. For the migration assay, $5 \times 10^{3}$ TLEC cells, treated with lentivirus or/and PD98059 were seeded in $100 \mu \mathrm{l}$ EBM-2 media with $1 \%$ FBS on the top of polyethylene terephthalate membranes within Transwell cell culture inserts (24-well inserts; pore size, $8 \mu \mathrm{m}$; Corning, Inc.). The bottom chamber was filled with $600 \mu \mathrm{l}$ EBM-2 media containing $2 \%$ FBS and $100 \mu 1$ supernatant from NIH3T3 cells (mouse embryonic fibroblast cell line) to act as a chemotactic factor. The cells were incubated for $48 \mathrm{~h}$ at $37^{\circ} \mathrm{C}$ with $5 \% \mathrm{CO}_{2}$. Subsequently, the cells were fixed in $2.5 \%(\mathrm{v} / \mathrm{v})$ glutaraldehyde for $20 \mathrm{~min}$ at room temperature and stained for $15 \mathrm{~min}$ with crystal violet at room temperature. Cells on the bottom were visualized under an inverted light microscope (Leica MicroSystems $\mathrm{GmbH}$ ) and were quantified by counting the number of cells in three randomly selected fields at a X100 magnification (Olympus Corporation).

Statistical analysis. Data are presented as the mean \pm SEM, and statistical analysis was performed using SPSS version 13.0 software (SPSS, Inc.). The experiments were repeated three times. Statistical comparisons were performed using one-way ANOVA followed by Tukey's post hoc test. $\mathrm{P}<0.05$ was considered to indicate a statistically significant difference.

\section{Results}

Separation of TLECs from a mouse xenograft cervical tumor model. Our previous study detected the co-localization of Sema4C and LYVE1 in human breast carcinoma (16). Firstly, the present study determined the expression level of Sema4C in TLECs in mouse cervical tumor tissue. Using a confocal microscope, LYVE1 was used to detect lymphatic vessels, and co-localization (merge) was identified between Sema4C (green) and LYVE1 (red; Fig. 1A). Primary TLECs were used to mimic the entire TME of cervical tumors, including the immune and proliferative conditions, as well as the interactions among cells, using C57BL/6 mice with U14 tumor xenografts. U14 cells were injected subcutaneously into the left shoulder, and LMVD was examined using LYVE1 immunohistochemistry (Fig. 1B). As determined via LMVD analysis, when the tumor grew to $1.0-1.5 \mathrm{~cm}^{3}$ [long diameter (length), 1.4-1.8 cm; short diameters (width), 1.2-1.8 cm], 1 week after injection, the lymphatic vessel density notably increased, compared with the 5 days group (Fig. 1C). After 14 days, massive necrosis occurred, and the density of lymphatic vessels was significantly decreased, compared with the 7 days group. Therefore, the appropriate time for the isolation of TLECs was considered to be day 7 after tumor cell injection. Moreover, LYVE1-positive cells were separated via flow cytometry (Fig. 1D). The cells were cultured successfully and appeared as a typical monolayer (Fig. 1E). VEGFR3 was used to identify the separated cells (Fig. 1F), and it was observed that the majority of the cells were positive for VEGFR3. Thus, the separated cells were positive for both LYVE1 and VEGFR3.

Sema4C regulates the migratory ability of TLECs. The expression levels of the target gene Sema4C were altered via lentiviral infection, and non-infected cells were used as blank controls (Fig. 2A). LV3-siRNA and full-length LV5-Sema4C, as well as the control lentiviruses LV3NC and LV5NC, all expressed green fluorescent protein (GFP) at a MOI of 40 at $72 \mathrm{~h}$ after transduction. The efficiency of the silencing lentivirus and the overexpression lentivirus infection was $\sim 90 \%$, according to GFP expression assessed via fluorescence microscopy. The qPCR results demonstrated a $\sim 70 \%$ reduction and a 3.5-fold increase in the Sema4C mRNA expression levels in the LV3-siRNA and LV5-Sema4C groups, respectively, compared with the control (Fig. 2B), both of which were statistically significant. Subsequently, the effects of Sema4C on the invasiveness of the TLECs were examined using a classical Transwell model. The 

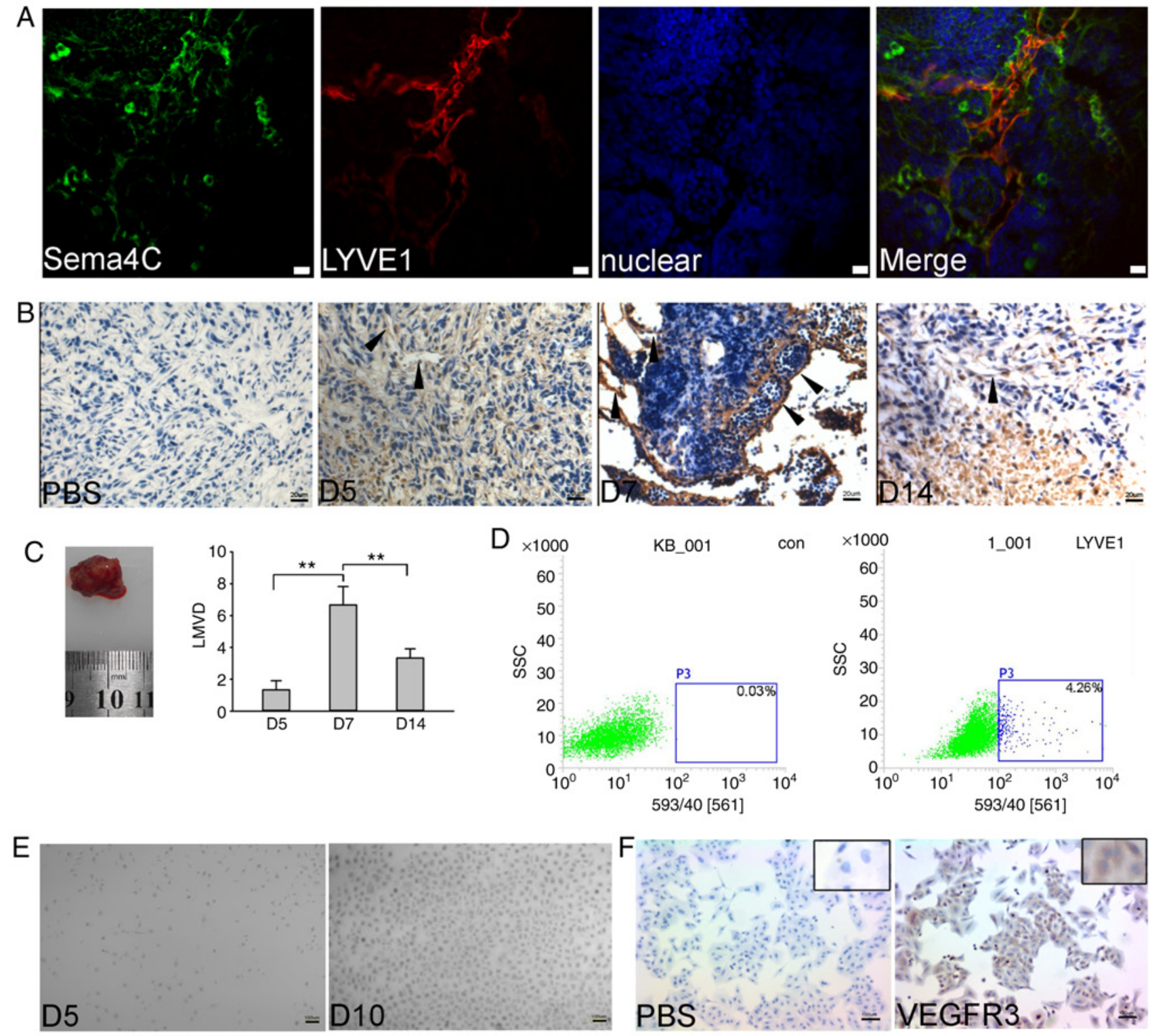

Figure 1. TLECs isolated from a mouse xenograft tumor. (A) Localization of Sema4C was detected in mouse tumor tissue. In lymphatic vessels, Sema4C (green) was co-localized with LYVE1 (red). Nuclei were identified via DAPI staining (blue). Scale bars, $20 \mu \mathrm{m}$. (B) LYVE1 immunohistochemical analysis indicated that day 7 after U14 cell injection was the most suitable time for isolation of TLECs. Arrowheads demonstrate TLEC positive staining. Scale bars, $20 \mu \mathrm{m}$. (C) Tumor image indicated the tumor size at day 7 after injection, and quantification of the numbers of lymphatic vessels on day 5, 7 and 14 was performed. (D) Tumor tissues were dissociated into a cell suspension and then stained for LYVE1-phycoerythrin. LYVE1-positive cells were separated via flow cytometry. The control group was used non-specific IgG. (E) TLECs were cultured in EBM-2 medium for 5 and 10 days. Scale bars, $10 \mu \mathrm{m}$. (F) VEGFR3-positive TLECs were identified via immunohistochemistry. Scale bars, $100 \mu \mathrm{m}$. The experiments were repeated three times. ${ }^{* *} \mathrm{P}<0.01$. TLECs, tumor-associated lymphatic endothelial cells; LYVE1, lymphatic vessel endothelial hyaluronan receptor 1; Sema4C, semaphorin 4C; LMVD, lymphatic microvessel density; SSC, side-scattered light; con, control.

number of cells at the bottom of the membrane, which reflects the migration of cells, was $56 \pm 9,52 \pm 8,51 \pm 6,40 \pm 8$ and $73 \pm 13$ for the control, LV3NC, LV5NC, LV3-siRNA and LV5-Sema4C groups, respectively. Cells at the bottom of the membrane were significantly reduced in the LV3-siRNA compared with the LV3NC group, and significantly increased in the LV5-Sema4C compared with the LV5NC group (Fig. 2C). Thus, silencing of Sema4C inhibited, and overexpression of Sema4C induced, the migratory ability of TLECs.

Sema $4 C$ regulates $E$-cadherin and ERK1/2 in TLECs. Based on the previously reported literature (10), a possible signaling pathway (Sema4C-E-caherin) underlying cell migration was investigated. It was revealed that Sema4C overexpression significantly inhibited the expression level of E-cadherin in the LV5-Sema4C group compared with LV5NC, whereas silencing of Sema4C significantly promoted its expression in the LV3-siRNA group compared with the LV3NC group. Moreover, the expression level of p-ERK1/2, a protein phosphorylation of ERK1/2 which constitutes the active form of ERK1/2, was significantly altered after lentiviral infection of the Sema4C full-length gene or Sema4C siRNA, compared with the LV5NC or LV3NC group, respectively. p-ERK could not be tested at the RNA level, so total ERK was tested at the RNA level to reveal the regulation of Sema4C (Fig. 3A). At the protein level, there were no significantly changes in 


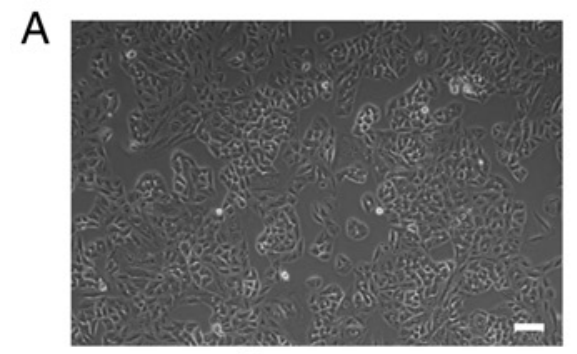

Con

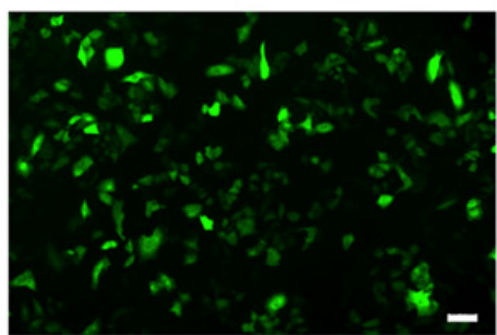

LV3-siRNA

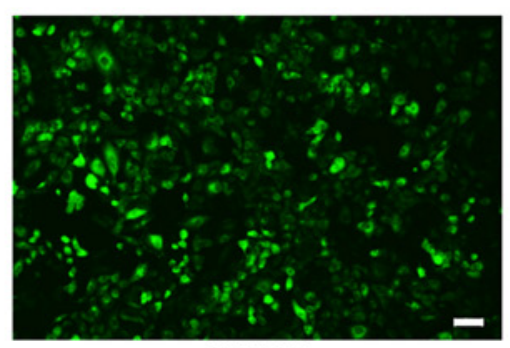

LV3NC

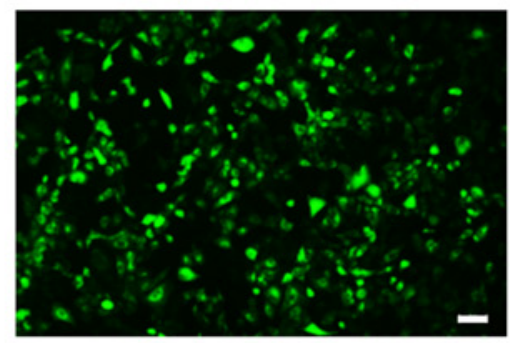

LV5-Sema4C

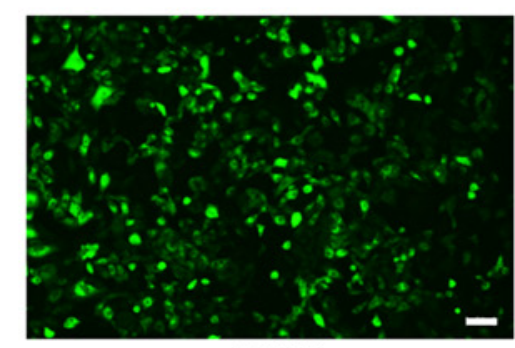

LV5NC

B
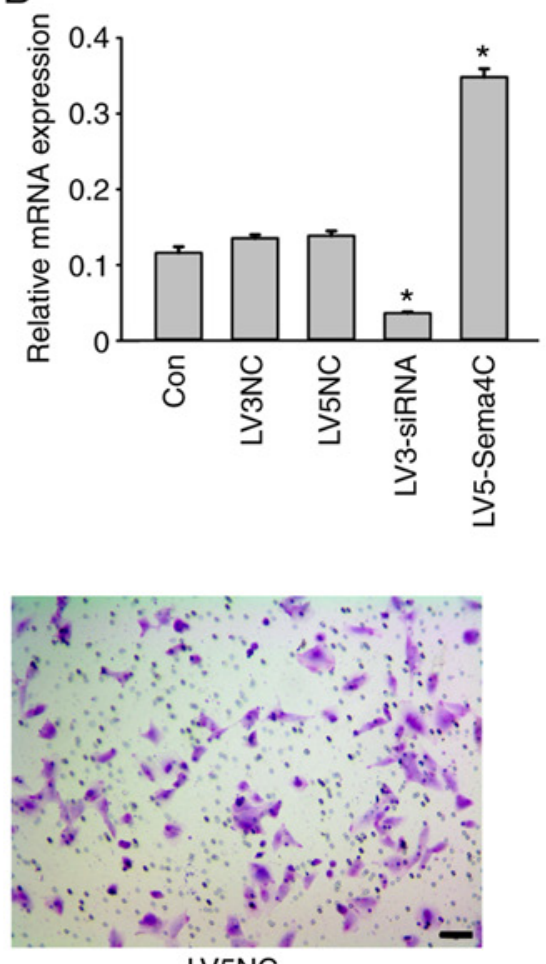

LV5NC

D

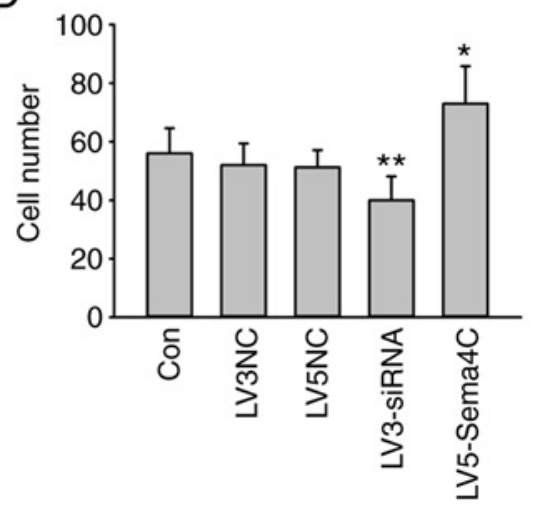

Figure 2. Role of Sema4C expression in the migratory ability of TLECs. (A) TLECs treated with lentiviral medium only, lentiviral control vector for Sema4C siRNA, lentiviral control vector for full-length Sema4C, Sema4C siRNA and full-length Sema4C. Scale bars, $10 \mu \mathrm{m}$. (B) TLECs with LV3-siRNA or LV5-Sema4C were generated, and Sema4C mRNA expression was measured via reverse transcription-quantitative PCR. (C) Migratory ability of cells was assessed using a Transwell assay. (D) Quantification of the number of cells at the bottom of the Transwell chamber. The experiments were repeated three times. ${ }^{*} \mathrm{P}<0.05,{ }^{* *} \mathrm{P}<0.01 \mathrm{LV} 3$-siRNA group vs. LV3NC group; LV5-Sema4C group vs. LV5NC group. Sema4C, semaphorin 4C; siRNA, small interfering RNA; $\mathrm{NC}$, negative control; TLECs, tumor-associated lymphatic endothelial cells.

total ERK (Fig. 3B and C). Therefore, it was indicated that Sema4C stimulated the phosphorylation of ERK. A similar pattern was observed for mRNA and protein expression levels of Sema4C and E-cadherin using RT-qPCR (Fig. 3A) and western blotting (Fig. 3B and C), respectively. Fig. 3D presents the p-ERK/ERK ratio in the five groups, and it was indicated that the activation of ERK was significantly reduced in the LV3-siRNA group compared with LV3NC, while it was significantly enhanced in the LV5-Sema4C group compared with LV5NC. 

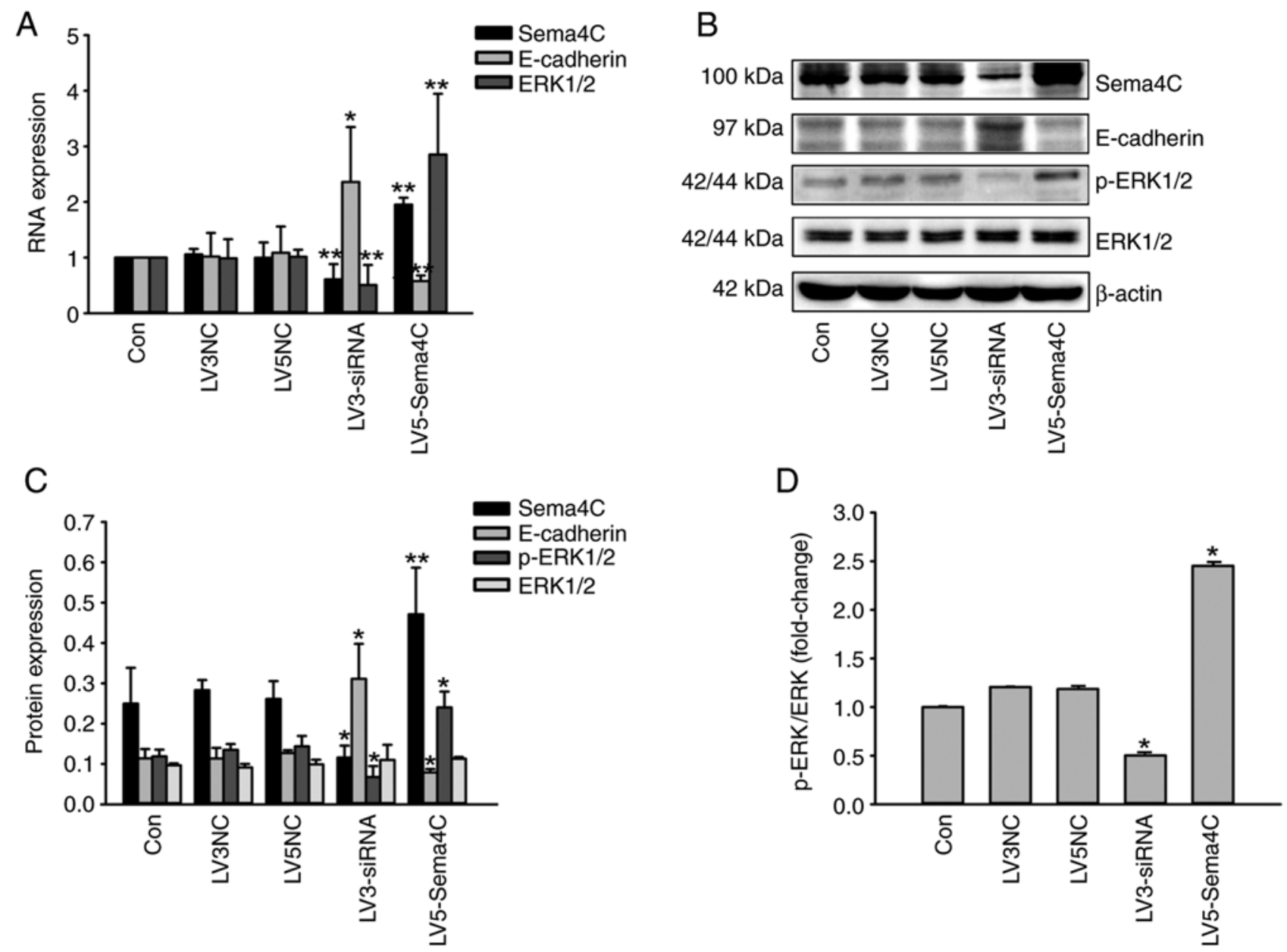

Figure 3. Role of Sema4C expression on E-cadherin and ERK1/2 expression of tumor-associated lymphatic endothelial cells. (A) Assessment of the mRNA expression levels of Sema4C, E-cadherin and ERK1/2 using reverse transcription-quantitative PCR. (B) Protein expression levels of Sema4C, E-cadherin, p-ERK1/2 and total ERK1/2 were detected using western blot analysis. (C) Semiquantitative analysis of protein expression levels of Sema4C, E-cadherin, p-ERK1/2 and total ERK1/2. (D) Expression of p-ERK1/2 was normalized to total ERK1/2, and represented as fold change over the control group. The experiments were repeated three times. "P<0.05 LV5-Sema4C group vs. LV5 NC group, ${ }^{* *} \mathrm{P}<0.01$ LV3-siRNA group vs. LV3NC group. Sema4C, semaphorin 4C; p, phosphorylated; siRNA, small interfering RNA; NC, negative control.

Sema4C regulates migration in TLECs via ERK activation. It was hypothesized that Sema4C regulated EndMT partially via the regulation of E-cadherin, which is one of the most important characteristics of EndMT. The ERK inhibitor PD98059 (30 mM for $24 \mathrm{~h}$ at room temperature) was employed to determine the alterations in the cell migratory ability following Sema4C silencing or overexpression via lentiviral infection. The results demonstrated that PD98059 enhanced the inhibition of cell migration induced by Sema4C siRNA compared with the control group (Fig. 4A). Furthermore, the addition of PD98059 reversed the promotion of TLEC migration induced following overexpression of Sema4C (Fig. 4A). The cell numbers at the bottom of the membrane, as presented in the bar graph, were $56 \pm 9,40 \pm 8,73 \pm 13,18 \pm 5$ and $58 \pm 9$ for control, LV3-siRN-, LV5-Sema4C, LV3-siRNA + PD98059 and LV5-Sema4C + PD98059 groups, respectively, reflecting the migration of the cells (Fig. 4B).

ERK inhibitor stimulates E-cadherin expression by blocking Sema $4 C$. The ERK inhibitor was used to determine whether the Sema4C-mediated regulation of E-cadherin was dependent on the phosphorylation level of ERK1/2. It was revealed that
Sema4C silencing significantly inhibited the phosphorylation level of ERK compared with control group (no treatment), and the introduction of PD98059 further enhanced the upregulation of E-cadherin expression induced by Sema4C siRNA infection, compared with the LV3-siRNA group. However, inhibition of the phosphorylation of ERK1/2 partially reversed the downregulation of E-cadherin that was mediated by Sema4C overexpression, compared with the LV5-Sema4C group. The observed mRNA expression levels of Sema4C and E-cadherin were in accordance with the protein expression levels (Fig. 5A, B and D-F). As the phosphorylation of ERK1/2 is indicative of the regulation of ERK1/2 at the protein level, the mRNA expression levels of ERK1/2 were also measured (Fig. 5C). Recovery of p-ERK1/2 expression was observed in the LV5-Sema4C + PD98059 group compared with the LV5-Sema4C group, indicating that PD98059 can effectively block the function of Sema4C (Fig. 5D and G). Moreover, ERK1/2 mRNA expression was significantly increased following Sema4C overexpression, but ERK1/2 protein expression did not change significantly, compared with the control group (Fig. 5C and $\mathrm{H}$ ), the alteration of p-ERK/ERK ratio showed the same alteration (Fig. 5I). Of note, the protein expression level of p-ERK1/2 exhibited the 

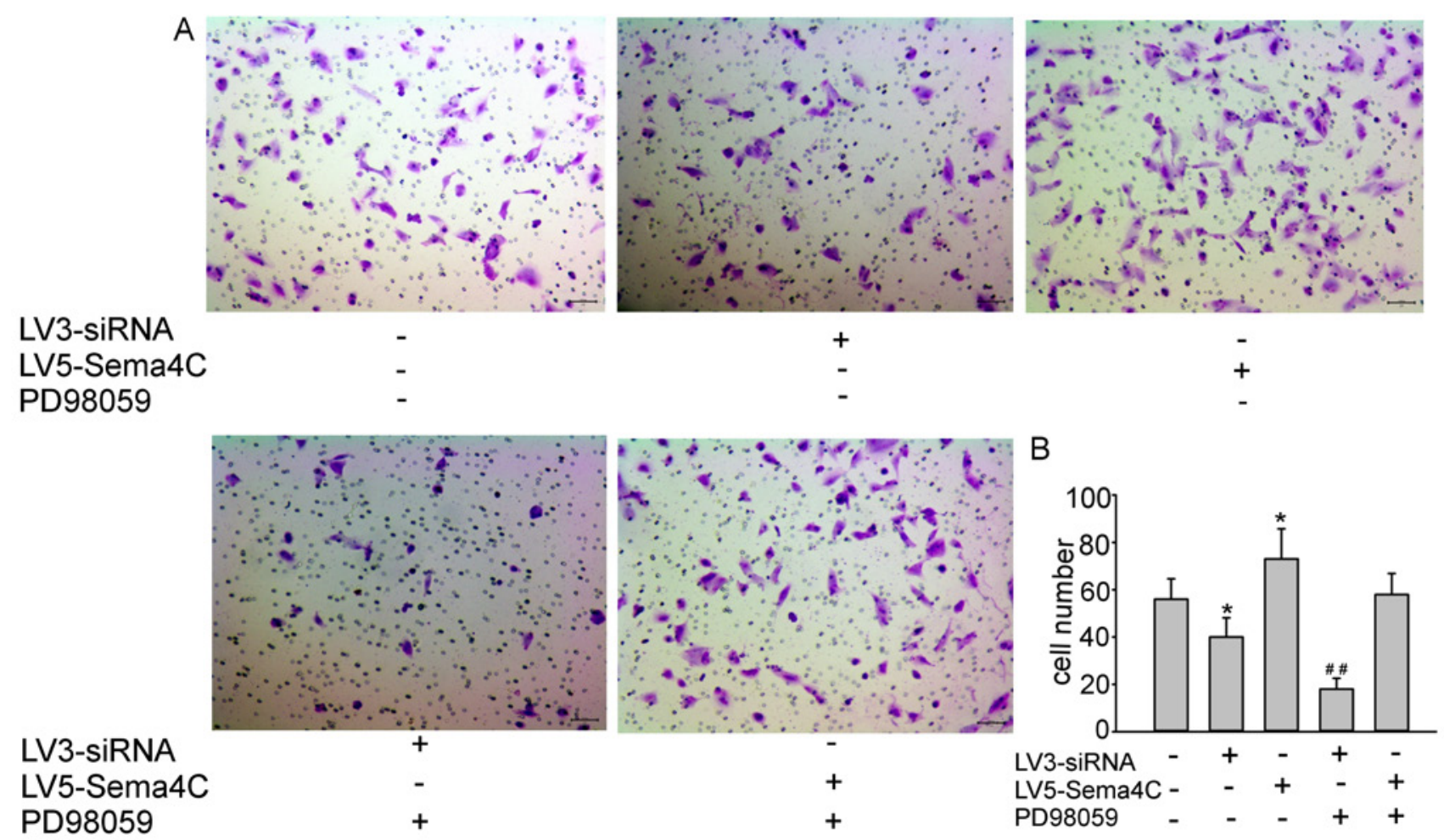

Figure 4. ERK inhibitor reverses effects on the migratory ability of TLECs induced by Sema4C. (A) Migratory ability of TLECs was assessed using cells treated with lentiviral medium only (control group), LV3-siRNA, LV5-Sema4C, LV3-siRNA + PD98059 and LV5-Sema4C + PD98059. Scale bars, 100 $\mu$ m. (B) Quantification of the number of cells from the bottom of the Transwell inserts was performed. The experiments were repeated three times. ${ }^{*}<0.05$ vs. control group; ${ }^{\#} \mathrm{P}<0.01$ vs. LV3-siRNA group. Sema4C, semaphorin 4C; siRNA, small interfering RNA; TLECs, tumor-associated lymphatic endothelial cells.

same tendency as the ERK1/2 mRNA expression. Thus, the phosphorylation level of ERK1/2 was an effective modification in the Sema4C signaling pathway.

\section{Discussion}

Tumor-associated lymphangiogenesis is a key modulator of tumor metastasis, although the underlying mechanism remains unknown. It was previously reported that the lymphatic system actively participates in tumor metastasis (23), and that LECs serve important roles in inducing immune tolerance (24). The ability of LECs to promote immunosuppression may induce tumor cell metastasis (24). A previous study using in situ laser capture microdissection revealed that the gene expression profile of human tumor LECs differed from that of normal LECs, and demonstrated that Sema4C was differentially expressed between LECs in tumor and normal tissues (16). The present study focused on the effect of LECs in attaining the cell invasive ability, which was attributed, at least in part, to the higher expression of Sema4C in tumor LECs.

There seem to be shared mechanisms between EMT and EndMT, and E-cadherin appeared in both of them $(5,25)$. It has been reported that EndMT occurs in cancer and tissue fibrosis (26), but the regulation of this process requires further investigation. In addition, whether and how LECs participate in EndMT to induce tumorous characteristics during cancer development is yet to be elucidated. The biological characteristics of LECs markedly change in tumors in vivo compared with normal tissues (17). Thus, in the present study, TLECs were isolated from mouse cervical tumor tissues via flow cytometry. LYVE1 was used as the marker for LEC separation (19), and VEGFR3 as an identification marker (27).

It has been reported that certain factors exhibit differences in their molecular mechanism between their membrane and soluble forms. For example, full-length Sema3C is a tumor angiogenesis inhibitor, whereas cleaved Sema3C is a tumor progression promoter (28). A previous study has revealed that biological properties differed between the soluble and membrane forms of Sema4C (sSema4C and mSema4C, respectively). For instance, sSema4C promoted lymphangiogenesis, whereas mSema4C directed cell-cell contacts, thereby providing the possibility of a new mechanism of mSema4C functioning in LECs (16). Therefore, the current study used lentiviral transfection for consistent overexpression or silencing of Sema4C in TLECs.

In a previous study, Ras homolog family member $\mathrm{A}$ (RhoA) was identified to be critical for Sema4C-mediated signaling (17). One of the distinct cell migration models, which is named amoeboid-type migration, is characterized by a spherical or elongated cell shape and is strongly dependent on Rho kinase activity (29). Thus, it was suggested that LECs migrate and undergo EndMT via amoeboid-type migration, which could be promoted by Sema4C, as indicated by the Transwell assay. A spherical cell shape with a small number of short protrusions was revealed in the cells after passing through the polyethylene terephthalate membranes (29). Wu et al (10) reported a p38 MAPK-dependent effect of Sema4C in terminal myogenic differentiation, including ERK regulation, indicating 

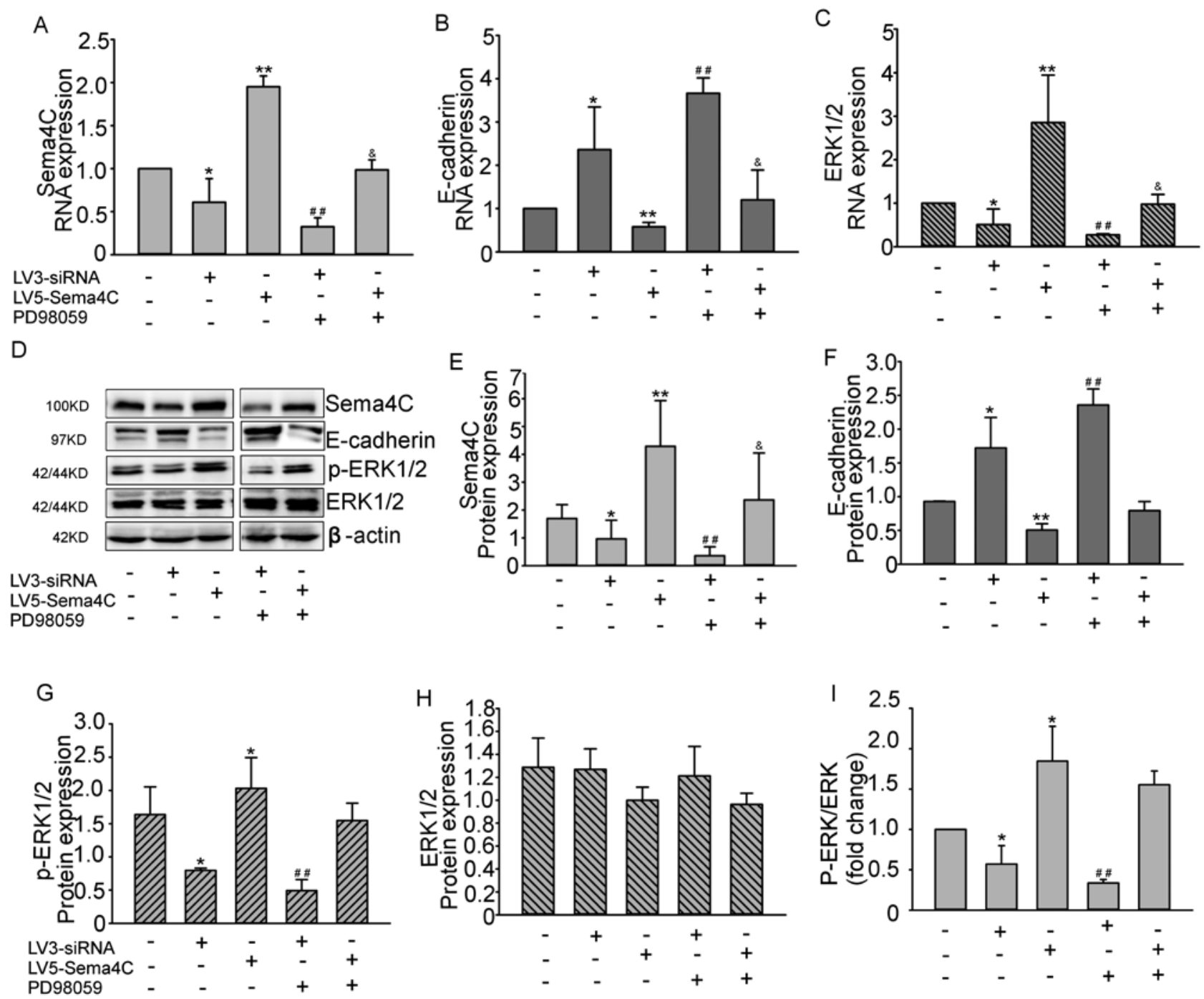

Figure 5. Sema4C regulates E-cadherin expression via the ERK pathway. mRNA expression levels of (A) Sema4C, (B) E-cadherin and (C) total ERK1/2 in TLECs treated with lentiviral medium only (control group), LV3-siRNA, LV5-Sema4C, LV3-siRNA + PD98059 and LV5-Sema4C + PD98059. (D) Protein expression levels of Sema4C, E-cadherin, p-ERK1/2 and total ERK1/2 in TLECs treated with lentiviral medium only, LV3-siRNA, LV5-Sema4C, LV3-siRNA + PD98059 and LV5-Sema4C + PD98059 detected using western blot analysis. Semiquantitative analysis of protein expression levels of (E) Sema4C, (F) E-cadherin, $(\mathrm{G})$ p-ERK1/2 and $(\mathrm{H})$ total ERK1/2. (I) Expression of p-ERK1/2 was normalized to total ERK1/2, and represented as fold change over the control group. The experiments were repeated three times. ${ }^{*} \mathrm{P}<0.05,{ }^{* *} \mathrm{P}<0.01$ vs. control group; ${ }^{\# \#} \mathrm{P}<0.01$ vs. LV3-siRNA group; ${ }^{\&} \mathrm{P}<0.05 \mathrm{LV} 5-\mathrm{Sema} 4 \mathrm{C}$ group. Sema4C, semaphorin 4C; p, phosphorylated; siRNA, small interfering RNA; TLECs, tumor-associated lymphatic endothelial cells.

a potential signaling pathway of Sema4C. The present study revealed that the promotion of the migratory ability of Sema4C-overexpressing LECs was in part attributed to ERK activation-induced repression of E-cadherin expression.

E-cadherin is a cell adhesive molecule that serves a key role in cellular adhesion and migration, constitutes one of the most important players in EndMT and can also be regulated by RhoA (30). Previous studies reported a novel role for E-cadherin in regulating LEC progeny in newly synthesized lymphatic vessels $(28,31)$. In particular, forced disruption of E-cadherin-mediated intercellular adhesion has been indicated to open the intercellular junctions in LEC monolayers, as determined by a previous study using Transwell assays (32). The present study demonstrated that repression of E-cadherin was an important molecular event for the promotion of the migratory ability in Sema4C-overexpressing LECs. This finding was in line with that of a previous study, which revealed that the overexpression of Sema4C suppressed E-cadherin, induced vimentin and promoted fibronectin secretion in human kidney cells (33). While the current results indicated that E-cadherin was an important molecular target of Sema4C in EndMT function, the downstream signaling pathways that were impacted by the loss of E-cadherin expression and promoted the cell migratory ability, along with the overexpression of Sema4C, are yet to be determined.

The expression of E-cadherin is regulated by an ERK-dependent mechanism $(34,35)$. Therefore, it will be interesting to determine whether the regulation of the Sema4C-mediated E-cadherin expression and migratory ability in LECs are activated by ERK. We focused on protein phosphorylation, a post-translational modification (PTM) with a prevalent role in the control of protein activity and signal transduction) (36). p-ERK could not be tested at the RNA level, so total ERK was tested at the RNA level to reveal the regulation of Sema4C in the present study. The application of an ERK inhibitor demonstrated that Sema4C 
regulated E-cadherin, and this process was dependent on ERK activation. It was revealed that the application of an ERK inhibitor could also affect the expression levels of Sema4C, and therefore, further studies are required to clarify whether other molecules are involved in this pathway and the feedback loop. Wei et al (16) revealed that Sema4C could be expressed in TLECs and promote the proliferation and migration of tumor cells by activating plexin-B2/MET signaling; however, the study by Wei et al (16) lacked molecular investigation in primary LECs. A previous study on primary lymphatic endothelial cells has demonstrated that there are differences between cultured cells and cells (37). Therefore, it is important to understand the molecular characteristic of primary TLECs.

Subsequent studies should focus on additional molecular markers of EndMT in LECs altered by Sema4C, as well as the cell morphology alterations, such as cytoskeletal changes, to demonstrate the role of Sema4C in regulating EndMT. Also, the therapeutic effect of lentivirus-mediated inhibition should be examined.

In conclusion, the present study demonstrated that Sema4C was expressed in TLECs via co-localization with LVYE1, and identified that upregulation of Sema4C was an important molecular mechanism that contributed to the induction of tumor-like characteristics by suppressing E-cadherin expression. Mechanistically, the process involved the activation of the ERK pathway, which functioned upstream of E-cadherin and downstream of Sema4C. Studies on TLECs are limited, and therefore, it will be important to determine whether enhanced ERK activation during the upregulation of Sema4C, as observed in TLECs, can induce tumor lymphatic metastasis, which in turn could be targeted using pharmacological approaches.

\section{Acknowledgements}

The authors would like to thank Dr Qingliang Wang (Department of Medical Affairs, Qilu Hospital, Cheeloo College of Medicine, Shandong University, Jinan, China) for his assistance with the statistical analysis of the present study.

\section{Funding}

The present study was supported by grants from the National Natural Science Foundation of China (grant nos. 81502238 and 81602286), the Department of Medical and Health Science Technology of Shandong Province (grant no. 2016w0345), the Department of Science Technology of Jinan City (grant no. 201705051), the China Postdoctoral Science Foundation (grant no. 2019T120594) and Natural Science Foundation General Project of Shandong Province (grant no. ZR2020MH230).

\section{Availability of data and materials}

The datasets used and/or analyzed during the current study are available from the corresponding author on reasonable request.

\section{Authors' contributions}

HW designed the experiments and wrote the paper. JP and XL conceived the experiments, performed the molecular experiments and analyzed the data. CL established the mouse model, performed the histological examination of tumor and separated the cells. MG performed the statistical analysis. HW and JP confirm the authenticity of all the raw data. All authors have read and approved the final manuscript.

\section{Ethics approval and consent to participate}

All the experiments were conducted in accordance with the National Institute of Health Guide of the Care and Use of Laboratory Animals. All the animal experimental protocols were approved by the Animal Care and Use Committee of Shandong University for Animal Ethics [approval no. KYLL-2015(KS)-079]. The use of primary LECs was approved by the Scientific Research Ethics Committee of Shandong University.

\section{Patient consent for publication}

Not applicable.

\section{Competing interests}

The authors declare that they have no competing interests.

\section{References}

1. Frumovitz M: Sentinel lymph node biopsy for cervical cancer patients-what's it gonna take? Gynecol Oncol 144: 3-4, 2017.

2. $\mathrm{Li} \mathrm{H}, \mathrm{Wu} X$ and Cheng $\mathrm{X}$ : Advances in diagnosis and treatment of metastatic cervical cancer. J Gynecol Oncol 27: e43, 2016.

3. Hoshino A and Lyden D: Metastasis: Lymphatic detours for cancer. Nature 546: 609-610, 2017.

4. Lucas ED and Tamburini BAJ: Lymph node lymphatic endothelial cell expansion and contraction and the programming of the immune response. Front Immunol 10: 36, 2019.

5. Saito A: EMT and EndMT: Regulated in similar ways? J Biochem 153: 493-495, 2013.

6. Kourtidis A, Lu R, Pence LJ and Anastasiadis PZ: A central role for cadherin signaling in cancer. Exp Cell Res 358: 78-85, 2017.

7. Wang SH, Chang JS, Hsiao JR, Yen YC, Jiang SS, Liu SH, Chen YL, Shen YY, Chang JY and Chen YW: Tumour cell-derived WNT5B modulates in vitro lymphangiogenesis via induction of partial endothelial-mesenchymal transition of lymphatic endothelial cells. Oncogene 36: 1503-1515, 2017.

8. Lee HR, Li F, Choi UY, Yu HR, Aldrovandi GM, Feng P, Gao SJ, Hong YK and Jung JU: Deregulation of HDAC5 by viral interferon regulatory factor 3 plays an essential role in Kaposi's sarcoma-associated herpesvirus-induced lymphangiogenesis. mBio 9: e02217-17, 2018.

9. Jiang L, Hao C, Li Z, Zhang P, Wang S, Yang S, Wei F and Zhang J: miR-449a induces EndMT, promotes the development of atherosclerosis by targeting the interaction between AdipoR2 and E-cadherin in Lipid Rafts. Biomed Pharmacother 109: 2293-2304, 2019.

10. Wu H, Wang X, Liu S, Wu Y, Zhao T, Chen X, Zhu L, Wu Y, Ding X, Peng X, et al: Sema4C participates in myogenic differentiation in vivo and in vitro through the p38 MAPK pathway. Eur J Cell Biol 86: 331-344, 2007.

11. Elder AM, Tamburini BAJ, Crump LS, Black SA, Wessells VM, Schedin PJ, Borges VF and Lyons TR: Semaphorin 7A promotes macrophage-mediated lymphatic remodeling during postpartum mammary gland involution and in breast cancer. Cancer Res 78: 6473-6485, 2018

12. Acker DWM, Wong I, Kang M and Paradis S: Semaphorin 4D promotes inhibitory synapse formation and suppresses seizures in vivo. Epilepsia 59: 1257-1268, 2018.

13. Sakurai A, Doci CL and Gutkind JS: Semaphorin signaling in angiogenesis, lymphangiogenesis and cancer. Cell Res 22: 23-32, 2012. 
14. Doci CL, Mikelis CM, Lionakis MS, Molinolo AA and Gutkind JS: Genetic identification of SEMA3F as an antilymphangiogenic metastasis suppressor gene in head and neck squamous carcinoma. Cancer Res 75: 2937-2948, 2015.

15. Nasarre P, Gemmill RM and Drabkin HA: The emerging role of class-3 semaphorins and their neuropilin receptors in oncology. Onco Targets Ther 7: 1663-1687, 2014.

16. Wei JC, Yang J, Liu D, Wu MF, Qiao L, Wang JN, Ma QF, Zeng Z Ye SM, Guo ES, et al: Tumor-associated lymphatic endothelial cells promote lymphatic metastasis by highly expressing and secreting SEMA4C. Clin Cancer Res 23: 214-224, 2017.

17. Albini A, Mirisola V and Pfeffer U: Metastasis signatures: Genes regulating tumor-microenvironment interactions predict metastatic behavior. Cancer Metastasis Rev 27: 75-83, 2008.

18. Olmeda D, Cerezo-Wallis D, Riveiro-Falkenbach E, Pennacchi PC, Contreras-Alcalde M, Ibarz N, Cifdaloz M, Catena X, Calvo TG, Cañón E, et al: Whole-body imaging of lymphovascular niches identifies pre-metastatic roles of midkine. Nature 546: 676-680, 2017

19. Young YK, Bolt AM, Ahn R and Mann KK: Analyzing the tumor microenvironment by flow cytometry. Methods Mol Biol 1458: 95-110, 2016

20. Lukacs-Kornek V: The role of lymphatic endothelial cells in liver injury and tumor development. Front Immunol 7: 548, 2016.

21. Faustino-Rocha A, Oliveira PA, Pinho-Oliveira J, Teixeira-Guedes C, Soares-Maia R, da Costa RG, Colaço B, Pires MJ, Colaço J, Ferreira R and Ginja M: Estimation of rat mammary tumor volume using caliper and ultrasonography measurements. Lab Anim (NY) 42: 217-224, 2013.

22. Livak KJ and Schmittgen TD: Analysis of relative gene expression data using real-time quantitative PCR and the 2(-Delta Delta C(T)) method. Methods 25: 402-408, 2001.

23. Humbert M, Hugues S and Dubrot J: Shaping of peripheral T cell responses by lymphatic endothelial cells. Front Immunol 7: 684, 2016.

24. Ji RC: Lymph nodes and cancer metastasis: New perspectives on the role of intranodal lymphatic sinuses. Int J Mol Sci 18: 51, 2016.

25. Potenta S, Zeisberg E and Kalluri R: The role of endothelial-to-mesenchymal transition in cancer progression. Br J Cancer 99: 1375-1379, 2008.

26. Cho JG, Lee A, Chang W, Lee MS and Kim J: Endothelial to mesenchymal transition represents a key link in the interaction between inflammation and endothelial dysfunction. Front Immunol 9: 294, 2018.
27. Harris AR, Perez MJ and Munson JM: Docetaxel facilitates lymphatic-tumor crosstalk to promote lymphangiogenesis and cancer progression. BMC Cancer 18: 718, 2018.

28. Mumblat Y, Kessler O, Ilan N and Neufeld G: Full-length semaphorin-3C Is an inhibitor of tumor lymphangiogenesis and metastasis. Cancer Res 75: 2177-2186, 2015.

29. Lawson CD and Ridley AJ: Rho GTPase signaling complexes in cell migration and invasion. J Cell Biol 217: 447-457, 2018.

30. Lee G, Kim HJ and Kim HM: RhoA-JNK regulates the E-cadherin junctions of human gingival epithelial cells. J Dent Res 95: 284-291, 2016.

31. Connor AL, Kelley PM and Tempero RM: Lymphatic endothelial lineage assemblage during corneal lymphangiogenesis. Lab Invest 96: 270-282, 2016.

32. Hou WH, Liu IH, Tsai CC, Johnson FE, Huang SS and Huang JS: CRSBP-1/LYVE-1 ligands disrupt lymphatic intercellular adhesion by inducing tyrosine phosphorylation and internalization of VE-cadherin. J Cell Sci 124: 1231-1244, 2011.

33. Zhou QD, Ning Y, Zeng R, Chen L, Kou P, Xu CO, Pei GC, Han $M$ and Xu G: Erbin interacts with Sema4C and inhibits Sema4C-induced epithelial-mesenchymal transition in HK2 cells. J Huazhong Univ Sci Technolog Med Sci 33: 672-679, 2013.

34. Marchese V, Juarez J, Patel P and Hutter-Lobo D: Densitydependent ERK MAPK expression regulates MMP-9 and influences growth. Mol Cell Biochem 456: 115-122, 2019.

35. Tang FY, Chiang EP, Chung JG, Lee HZ and Hsu CY: S-allylcysteine modulates the expression of E-cadherin and inhibits the malignant progression of human oral cancer. J Nutr Biochem 20: 1013-1020, 2009.

36. Kawata K, Yugi K, Hatano A, Kokaji T, Tomizawa Y, Fujii M, Uda S, Kubota H, Matsumoto M, Nakayama KI and Kuroda S: Reconstruction of global regulatory network from signaling to cellular functions using phosphoproteomic data. Genes Cells 24: 82-93, 2019.

37. Wick N, Saharinen P, Saharinen J, Gurnhofer E, Steiner CW, Raab I, Stokic D, Giovanoli P, Buchsbaum S, Burchard A, et al: Transcriptomal comparison of human dermal lymphatic endothelial cells ex vivo and in vitro. Physiol Genomics 28: 179-192, 2007.

(i) $($ This work is licensed under a Creative Commons Attribution-NonCommercial-NoDerivatives 4.0 International (CC BY-NC-ND 4.0) License. 